\title{
TUBERCULOSIS CONGÉNITA PRE-EXTENSIVAMENTE RESISTENTE A DROGAS: REPORTE DE CASO
}

\author{
Hernán del Castillo ${ }^{1, a}$, Antonio Salas Lopez $2, a$, Anna Paredes Temoche ${ }^{3, b}$, Alvaro Soto Mosquera ${ }^{3, b}$, \\ Tania Valerio Rojas ${ }^{3, b}$
}

\begin{abstract}
RESUMEN
La tuberculosis en el lactante es un cuadro de difícil diagnóstico por las pruebas diagnósticas que muchas veces resultan negativas y por la dificultad de identificar la fuente de transmisión. Se presenta el caso de un lactante varón de un mes de vida que presenta irritabilidad, taquipnea, fiebre, pobre ganancia de peso desde el nacimiento y hepatomegalia, además, tiene el antecedente materno de tuberculosis pre-extensivamente resistente a drogas y reacción granulomatosa tuberculoide con tinción auramina positiva para bacilos ácido-alcohol resistentes en la histopatología de placenta. Ante la sospecha de tuberculosis congénita, es referido al Instituto Nacional de Salud del Niño para estudio diagnóstico y tratamiento; el paciente presenta una evolución clínica favorable y sin reacciones adversas al tratamiento. El diagnóstico de tuberculosis congénita debe considerarse en lactantes con signos clínicos sugestivos de la enfermedad y mantener la sospecha ante la presencia del antecedente materno de infección por Mycobacterium tuberculosis.
\end{abstract}

Palabras clave: Mycobacterium tuberculosis; Tuberculosis Extensivamente Resistente a Drogas; Transmisión Vertical de Enfermedad Infecciosa (fuente: DeCS BIREME)

\section{PRE-EXTENSIVELY DRUG-RESISTANT CONGENITAL TUBERCULOSIS: CASE REPORT}

\begin{abstract}
Tuberculosis in infants is a clinical case difficult to diagnose by regular testing which often yield negative results; additionally, the source of transmission is difficult to identify. This work presents the case of a one-month old nursing boy presenting irritability, tachypnea, fever, poor gain weight from birth, and hepatomegaly. Additionally, he had the maternal history of pre-extensively drug- resistant tuberculosis and tuberculoid granulomatosis reaction with positive auramine tincture for acid-alcohol resistant bacilli at histopathology of the placenta. With a suspected congenital tuberculosis, he was referred to the National Children's Health Institute for diagnosis and treatment. The patient showed a favorable clinical evolution and no adverse reactions to treatment. The diagnosis of congenital tuberculosis must be considered in infants with suggestive clinical signs of the disease and such suspicion must be maintained with the presence of a maternal history of Mycobacterium tuberculosis infection.
\end{abstract}

Keywords: Mycobacterium tuberculosis; Extensively Drug-Resistant Tuberculosis; Vertical Transmission of Infectious Disease (source: MeSH NLM)

\section{INTRODUCCIÓN}

La tuberculosis (TB) congénita es una condición que ocurre como resultado de la transmisión de la TB de la madre al feto. Es considerada una de las infecciones congénitas menos frecuentes, con pocos casos reportados en la literatura médica ${ }^{(1-3)}$; sin embargo, no debe ser desestimada debido a la alta tasa de mortalidad que puede llegar hasta un $50 \%{ }^{(4)}$.

Existen dos mecanismos de infección en el neonato: el primero es por vía hematógena donde los bacilos atraviesan la barrera placentaria y viajan a través del cordón umbilical hacia el hígado, para formar un complejo granulomatoso primario, secundariamente el pulmón u otros órganos serán infectados desencadenando una respuesta inflamatoria sistémica entre la segunda y cuarta semana de vida; el segundo modo de trasmisión es mediante el contacto directo del neonato con las paredes lesionadas del canal de parto o por aspiración del líquido amniótico ${ }^{(5)}$.

Para el diagnóstico de esta entidad, en 1994 Cantwell et al. propusieron criterios de utilidad clínica vigentes hasta la actualidad; para ello, el niño debe presentar lesiones

\footnotetext{
Instituto Nacional de Salud del Niño. Lima, Perú

Hospital Nacional Dos de Mayo. Lima, Perú

Sociedad Científica de San Fernando, Universidad Nacional Mayor de San Marcos. Lima, Perú

Médico neumólogo; ${ }^{\mathrm{b}}$ estudiante de Medicina

Recibido: 01/05/2018 Aprobado: 24/10/2018 En línea: 21/12/2018
}

Citar como: del Castillo H, Salas Lopez A, Paredes Temoche A, Soto Mosquera A, Valerio Rojas T. Tuberculosis congénita pre-extensivamente resistente a drogas: reporte de caso. Rev Peru Med Exp Salud Publica. 2018;35(4):695-8. doi:10.17843/rpmesp.2018.354.3647 
tuberculosas y al menos uno de los siguientes condiciones: las lesiones deben aparecer en la primera semana de vida; debe observarse un complejo hepático primario o granulomas caseificantes hepáticos; hallazgo de infección tuberculosa en la placenta o tracto genital de la madre; o exclusión de la posibilidad de transmisión posnatal a través de una investigación de contactos ${ }^{(6)}$.

No obstante, el diagnóstico temprano es difícil debido a la clínica variable y atípica que puede presentarse, lo cual retrasa el diagnóstico y empeora el pronóstico ${ }^{(1,4,6)}$. Presentamos un caso de TB congénita en un recién nacido varón con bajo peso y fiebre, cuya madre tiene como antecedente TB pre-extensivamente resistente a drogas (XDR), definida como una cepa de TB MDR resistente a una fluoroquinolona o inyectable de segunda línea, pero no a ambos ${ }^{(7)}$.

\section{REPORTE DE CASO}

Como antecedente podemos mencionar que, la madre de profesión técnica en enfermería, fue diagnosticada de TB pulmonar sensible en 2009, con abandono de tratamiento luego de dos meses. En febrero de 2017, con 27 años de edad, reinicia tratamiento para TB sensible el cual nuevamente descontinua luego de 45 días. En septiembre del 2017 durante su séptimo mes de gestación acude al hospital por presentar un cuadro de disnea, tos, dolor torácico y disfonía. Luego de los estudios correspondientes, es diagnosticada con TB pre-XDR, multisistémica, que comprometió laringe y pulmones.

Se descartó infección por virus de inmunodeficiencia humana. Su prueba de sensibilidad convencional en agar en placa reveló resistencia a rifampicina, isoniacida, etambutol, estreptomicina, kanamicina y capreomicina; y demostró sensibilidad a pirazinamida, etionamida, levofloxacino y cicloserina. En relación con el mencionado patrón de sensibilidad, recibió tratamiento individualizado consistente en pirazinamida, imipenem/cislastatina, linezolid, amoxicilina/ ácido clavulánico, moxifloxacino y clofazimina con evolución favorable.

El nacimiento del menor de sexo masculino fue pretérmino y programada por cesárea por factor de riesgo materno de TB pre-XDR. Al examen físico se evidenció leve tiraje subcostal e hígado palpable a $2,5 \mathrm{~cm}$ debajo del reborde costal derecho. Los exámenes de laboratorio al nacimiento mostraron una hemoglobina de $20,3 \mathrm{~g} / \mathrm{dL}$; leucocitos de 16 250/mm ${ }^{3}, 2 \%$ de abastonados, $47 \%$ de neutrófilos; plaquetas de $284000 / \mathrm{mm}^{3}$, cultivo de líquido cefalorraquídeo (LCR) y Proteína $C$ reactiva (PCR) para Mycobacterium tuberculosis en LCR negativos, baciloscopía de orina, heces y aspirado gástrico negativos. También se le realizó una prueba de Mantoux, que resultó negativa. Sin embargo, la histopatología de placenta demostró reacción granulomatosa tuberculoide con tinción con auramina positiva para bacilos ácido-alcohol resistentes. Fue dado de alta sin intercurrencias antes del primer mes de vida.
Al mes de vida, el paciente es llevado a un hospital de tercer nivel por presentar irritabilidad, taquipnea y fiebre de $38^{\circ} \mathrm{C}$, al examen físico se encontró pobre ganancia de peso de (566 $\mathrm{g}$ desde el nacimiento) y se palpó hígado a $3 \mathrm{~cm}$ debajo del reborde costal derecho, se hospitalizó con diagnóstico de sepsis de probable punto de partida urinario. Los exámenes de laboratorio mostraron una hemoglobina de $6,8 \mathrm{~g} / \mathrm{dL}$; leucocitos de $35740 / \mathrm{mm}^{3}, 6 \%$ de abastonados, $83 \%$ de neutrófilos y plaquetas de $550000 / \mathrm{mm}^{3}$, en el examen de orina se observó 141 leucocitos/campo, 3 hematíes/campo, $\mathrm{pH} 6$. Se confirmó hepatomegalia con ecografía abdominal y con radiografía de tórax, se informó además infiltrado retrocardiaco izquierdo (Figura 1), fue manejado con meropenem $100 \mathrm{mg} / \mathrm{Kg} / \mathrm{d}$ por 13 días y vancomicina $40 \mathrm{mg} / \mathrm{Kg} / \mathrm{d}$ por 11 días.

Debido al antecedente de la madre, la anomalía radiográfica, hepatomegalia y los signos sugerentes de infección se sospecha de TB congénita, el paciente es referido al Instituto Nacional de Salud del Niño (INSN) para estudio diagnóstico y tratamiento.

En el INSN el menor es recibido con un peso de $2850 \mathrm{~g}$ y una temperatura de $37,5^{\circ} \mathrm{C}$, hígado palpable a $2 \mathrm{~cm}$ debajo del reborde costal derecho, no presentaba otros hallazgos de importancia.

Se le realizan nuevamente exámenes de laboratorio encontrando una hemoglobina de $11 \mathrm{~g} / \mathrm{dL}$, leucocitos en $15750 / \mathrm{mm}^{3}, 2 \%$ de eosinófilos, $32 \%$ de segmentados, $52 \%$ de linfocitos y 557000 plaquetas $/ \mathrm{mm}^{3}$, el urocultivo fue negativo y el resto de exámenes de laboratorio fueron normales. También se le realizó una tomografía de tórax (Figura 2), donde se evidencian micronódulos en bases pulmonares. Además, se realizaron cultivo de aspirado gástrico, baciloscopías de orina, heces y aspirado gástrico, los cuales fueron negativos.

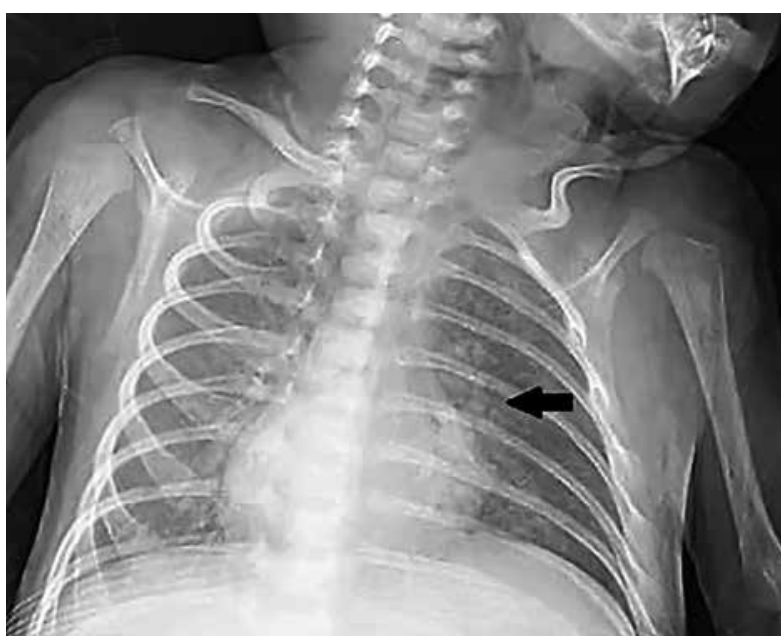

Figura 1. Radiografía de tórax anteroposterior donde se evidencia un infiltrado retrocardiaco en pulmón izquierdo (flecha negra) 


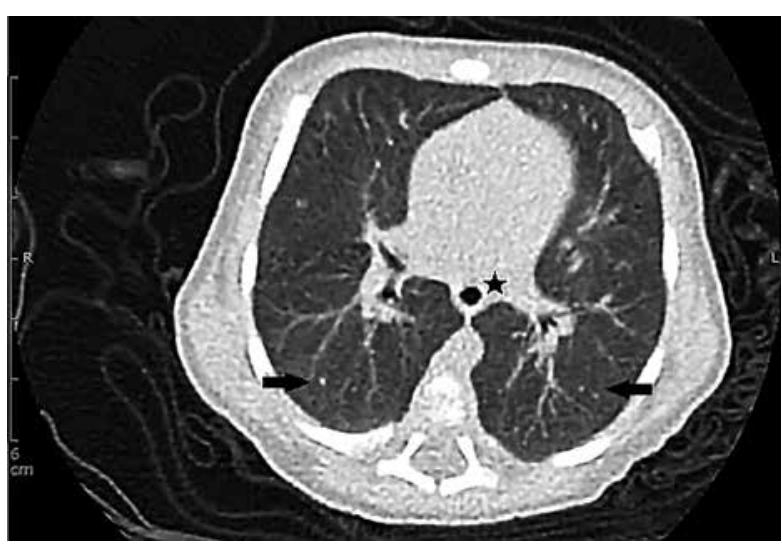

Figura 2. Tomografía axial de tórax sin contraste, donde se señala (flechas negras) la presencia de micronódulos en ambas bases pulmonares y un discreto patrón intersticial en base derecha. Se encuentra esófago dilatado (estrella)

Debido al antecedente materno y a la tomografía con hallazgos anormales, se decide iniciar tratamiento para TB basado en el esquema de la madre, consistente en moxifloxacino $10 \mathrm{mg} /$ $\mathrm{kg} / \mathrm{d}$, meropenem $94,7 \mathrm{mg} / \mathrm{kg}$, linezolid $17,5 \mathrm{mg} / \mathrm{kg} / \mathrm{d}$, amoxicilina/Ácido clavulánico $80,7 \mathrm{mg} / \mathrm{kg} / \mathrm{d}$, cicloserina 15 $\mathrm{mg} / \mathrm{kg} / \mathrm{d}$, etionamida $15 \mathrm{mg} / \mathrm{kg} / \mathrm{d}$ y pirazinamida $29,8 \mathrm{mg} / \mathrm{kg} / \mathrm{d}$. Adicionalmente se le indicó piridoxina $2,8 \mathrm{mg} / \mathrm{kg} / \mathrm{d}$ (por el uso de cicloserina, que aumenta el aclaramiento de la vitamina B6). A la fecha del reporte, lleva seis meses de tratamiento. Para la administración de las drogas por vía endovenosa se le realizó un acceso venoso central de larga duración (catéter Port).

Con el fin de monitorizar los posibles efectos adversos al tratamiento se solicitaron hemogramas completos (para descartar la posibilidad de mielosupresión por linezolid), electrocardiograma (para evaluar la posibilidad de QT alargado por moxifloxacino), pruebas de función hepática (por la pirazinamida) y renal, electrolitos séricos, así como una prueba de potenciales evocados auditivos (por la posibilidad de alteraciones electrolíticas, nefrotoxicidad y ototoxicidad de la amikacina, que fue parte del tratamiento inicial de la madre durante la gestación, antes de contar con su prueba de sensibilidad convencional, tras la cual se suspendió. Se realizaron baciloscopías de control de orina, heces y aspirado gástrico que fueron negativas. Actualmente el tratamiento continúa y la evolución es favorable.

\section{DISCUSIÓN}

La TB congénita es una forma de presentación rara de la infección tuberculosa, cuya transmisión se da intraútero. Usualmente el diagnóstico de la madre se hace posparto, debido a que suelen ser asintomáticas durante el embarazo ${ }^{(2)}$, siendo la activación de la enfermedad durante la gestación un factor de riesgo importante para la trasmisión vertical $^{(8)}$.
La histología placentaria puede revelar reacción granulomatosa o no ${ }^{(5,9)}$, una explicación para la ausencia de dichos hallazgos es la focalidad de la lesión y el tiempo de evolución ${ }^{(9)}$. En el presente caso, se resalta el hallazgo de la reacción granulomatosa en la placenta, además del antecedente materno que motivó la sospecha de TB congénita.

La clínica de la TB congénita es inespecífica y variada, haciendo que sea fácil confundirla con otras infecciones congénitas o neonatales, y suele aparecer luego de la tercera semana de vida ${ }^{(6)}$. Los signos más frecuentes son hepatoesplenomegalia, dificultad respiratoria, fiebre y linfadenopatías ${ }^{(1)}$. Otras características clínicas pueden incluir distensión abdominal, letargia, dermatopatías, ictericia, otorrea y anomalías hematológicas ${ }^{(3)}$. En nuestro caso se destaca la fiebre, irritabilidad, hepatomegalia y pobre ganancia de peso como sintomatología sugestiva y concordante con la literatura. Sin embargo, la leucocitosis en orina inclinó la sospecha hacia un proceso infeccioso urinario, lo cual retrasó el diagnóstico.

Para el diagnóstico de infección, la prueba de tuberculina (Mantoux) puede tardar hasta tres meses en volverse positiva ${ }^{(6,10)}$, es más, se encuentra positiva en menos del $15 \%$ de los casos y se han descritos casos confirmados de TB congénita en los cuales fue negativa ${ }^{(5)}$, al igual que en el presente reporte.

Respecto a las imágenes, las radiografías de tórax de los infantes con TB congénita varían desde normales hasta hallazgos patológicos como patrón miliar, consolidación, cavitación, patrones intersticiales, nódulos múltiples, y linfadenopatías hiliares y mediastinales, entre los más frecuentes ${ }^{(1,2,8)}$. La tomografía de tórax es más sensible para detectar algunos de estos signos, como en el presente reporte, donde se halló en un inicio, un infiltrado retrocardiaco izquierdo y luego se evidenció la presencia de micronódulos en ambas bases pulmonares con discreto patrón reticular.

La TB en este grupo de edad es de difícil diagnóstico por las múltiples presentaciones clínicas ${ }^{(1,4,6)}$, por las pruebas diagnósticas que muchas veces salen negativas y por la dificultad de identificar la fuente de transmisión. En el presente caso se consideró el diagnóstico de TB congénita por el infiltrado radiológico y tomográfico sugerente de TB, por las lesiones granulomatosas con tinción positiva para bacilos ácido-alcohol resistentes en la placenta, por el descarte de contactos en la etapa posnatal del lactante y por el antecedente esencial de la madre.

En cuanto al tratamiento, no existen guías nacionales estandarizadas para tratar la TB-MDR congénita o TB-XDR congénita, lo cual dificulta la aplicación de nuevos esquemas, vías, dosis y tiempo de duración individualizados, además, la presencia de efectos adversos frecuentemente obliga a cambiar los esquemas establecidos ${ }^{(11)}$. 
Entre los efectos adversos más importantes se encuentran la hepatotoxicidad por isoniazida, rifampicina y pirazinamida ${ }^{(12)}$, alteración del intervalo QT en el electrocardiograma por quinolonas ${ }^{(13)}$, mielosupresión por linezolid ${ }^{(14)}$ y nefrotoxicidad, ototoxicidad y alteraciones electrolíticas por aminoglucósidos inyectables (15). Por ello, el manejo y seguimiento deben ser siempre acompañados de exámenes que permitan valorar la posibilidad de que se esté presentando efectos adversos. En el presente reporte se realizaron los exámenes correspondientes, no hallándose ningún evento adverso que motivara el cambio de algún medicamento.

Como limitación del presente reporte debemos indicar que no se pudo obtener una prueba diagnóstica microbiológica confirmatoria. Se realizaron los exámenes pertinentes, sin embargo, estos fueron negativos. A pesar de ello, la epidemiología, cuadro clínico, hallazgos radiográficos e histopatológicos de la placenta fueron justificación suficiente para iniciar tratamiento.

Concluimos que, ante un cuadro clínico inespecífico con signos sugerentes de TB, acompañado de factores epidemiológicos favorables, nuestra sospecha diagnóstica debería ser TB; incluso si las pruebas microbiológicas resultan negativas, no debería ser un impedimento para iniciar tratamiento.

Criterios éticos: Se informó a la madre del paciente la intención de publicar el presente reporte, obteniéndose el consentimiento informado requerido y los permisos institucionales respectivos.

Contribuciones de autoría: HDC, ASL, APT, ASM y TVR han participado en la concepción y diseño del artículo; APT, ASM y TVR en la recolección de resultados y redacción del artículo. Además, HDC y ASL contribuyeron con el análisis e interpretación de datos, revisión crítica del artículo y aprobación de la versión final.

Conflictos de intereses: Los autores declaran no tener conflictos de intereses en la publicación del presente caso.

Fuentes de financiamiento: autofinanciado

Conflictos de interés: los autores declaran no tener conflictos de interés

\section{REFERENCIAS BIBLIOGRÁFICAS}

1. Chang C, Wu PW, Yeh C, Wong K, Wang J, Chang C. Paediatrics and International Child Health Congenital tuberculosis: case report and review of the literature. Paediatr Int Child Health. 2017;9047:1-4. doi: 10.1080/20469047.2017.1315912.

2. Peng W, Yang J, Liu E. Analysis of 170 cases of congenital TB reported in the literature between 1946 and 2009. Pediatr Pulmonol. 2011;46(12):1215-24.

3. Sáenz-Gómez J, Karam Bechara J, Jamaica Balderas L. Tuberculosis perinatal. Bol Med Hosp Infant Mex. 2015;72(1):61-5. doi: 10.1016/j.bmhimx.2015.01.009.

4. Whittaker E, Kampmann B. Perinatal tuberculosis. New challenges in the diagnosis and treatment of tuberculosis in infants and the newborn. Early Hum Dev. 2008;84(12):795-9. doi: 10.1016/j.earlhumdev.2008.09.005.

5. Dewan P, Gomber S, Das S. Congenital tuberculosis: a rare manifestation of a common disease. Paediatr Int Child Health. 2014;34(1):60-2. doi: 10.1179/2046905513Y.0000000051.

6. Cantwell MF, Shehab ZM, Costello AM, Sands L, Green WF, Ewing EP, et al. Congenital Tuberculosis. N Engl
J Med. 1994;330(15):1051-4. doi: 10.1056/NEJM199404143301505.

7. Mirza IA, Khan FA, Khan KA, Satti L, Ghafoor T, Fayyaz M. Extensively and pre-extensively drug resistant tuberculosis in clinical isolates of multi-drug resistant tuberculosis using classical second line drugs (levofloxacin and amikacin). J Coll Physicians Surg Pak. 2015;25(5):337-41.

8. Saramba MI, Zhao D. A Perspective of the Diagnosis and Management of Congenital Tuberculosis. J Pathog. 2016;2016:8623825. doi: 10.1155/2016/8623825.

9. Abramowsky CR, Gutman J, Hilinski JA. Mycobacterium Tuberculosis Infection of the Placenta: A Study of the Early (Innate) Inflammatory Response in Two Cases. Pediatr Dev Pathol. 2012;15(2):132-6. doi: 10.2350/1105-1039-CC.1.

10. Mora-Bautista VM, Cala-Vecino LL. Tuberculosis congénita, tras un enemigo oculto. Infectio. 2016;20(3):172-5. doi: $10.1016 /$ j.infect.2015.09.003.

11. Palacios E, Dallman R, Muñoz M, Hurtado R, Chalco K, Guerra D, et al. Drug-Resistant Tuberculosis and Pregnancy: Treatment Outcomes of 38 Cases in Lima, Peru. Clin Infect Dis. 2009;48(10):1413-9. doi: $10.1086 / 598191$
12. Nagarajan S, Whitaker P. Management of adverse reactions to first-line tuberculosis antibiotics. Curr Opin Allergy Clin Immunol. 2018;18(4):333-341. doi: 10.1097/ACI.0000000000000462.

13. Yoon H-Y, Jo K-W, Nam GB, Shim TS. Clinical significance of QT-prolonging drug use in patients with MDR-TB or NTM disease. Int J Tuberc Lung Dis. 2017;21(9):996-1001. doi: 10.5588/ ijtld.17.0174.

14. Ballesteros García MDM, Orós Milián ME, Díaz Velázquez E, Alarcón Alacio MT, Galán Del Río P. Mielosupresión inducida por linezolid: un caso pediátrico. Arch Argent Pediatr. 2017;115(6):e420-e423. doi: 10.5546/aap.2017.eng.e420.

15. Garcia-Prats AJ, Schaaf HS, Hesseling AC. The safety and tolerability of the second-line injectable antituberculosis drugs in children. Expert Opin Drug Saf. 2016;15(11):1491-1500. doi: 10.1080/14740338.2016.1223623.

Correspondencia: Alvaro Soto Mosquera Dirección: Sector 2, Grupo 8, Manzana B, Lote 23. Villa el Salvador. Lima, Perú Teléfono: (511) 977139919

Correo electrónico: soto22alvaro@gmail.com 